\title{
Representação e Classificação de Texturas da Íris Baseada na Transformada Ótima de Gabor
}

\author{
Fernanda Rispoli Quartieri ${ }^{1}$ \\ Jacob Scharcanski ${ }^{1}$ \\ Letícia Vieira Guimarães $^{1}$ \\ Adalberto Schuck Junior ${ }^{1}$
}

Resumo: Este trabalho propõe a aplicação e investigação do método apresentado por Manjunah e Ma [12] para análise de texturas e denominado de Transformada Wavelet Gabor Ótima (TWGO) na representação e discriminação de texturas da íris. $\mathrm{O}$ método proposto foi testado usando um banco de 1205 imagens de olhos referenciado na literatura [16]. Em cada uma destas imagens, a região da íris foi segmentada e após, representada em múltiplas escalas segundo a TWGO; os padrões de textura são então representados por estatísticas, e comparados entre si através de medidas de similaridade para fazer a identificação da íris. Os resultados de diversos testes são apresentados, e indicam que o método proposto produz elevadas taxas de acerto no reconhecimento da íris (94,68\% de acerto considerando imagens fora de foco e com oclusões da íris, e 100\% excluindo estas imagens problemáticas). $\mathrm{O}$ método proposto permite ajustar a sensibilidade do critério de similaridade entre texturas de acordo com o nível de segurança requerido pela aplicação.

\begin{abstract}
This paper proposes to investigate the application of the method developed by Manjunah and Ma [12], namely, the Optimal Gabor Wavelet Transform (OGWT), in the context of iris texture representation. The proposed method was tested in a widely known database of 1205 eye images [16]. In each one of these images, the iris region was segmented, and then represented in multiple scales using the OGWT; the íris texture patterns were represented by their statistics in the Wavelet domain, and compared using similarity metrics. The experimental results indicate that the proposed
\end{abstract}

1 Programa de Pós-Graduação em Engenharia Elétrica - PPGEE, Universidade Federal do Rio Grande do Sul - UFRGS, Av. Oswaldo Aranha, 103, Porto Alegre, RS, Brasil \{fernanda.quartieri@ufrgs.br, schuck@ufrgs.br, jacobs@inf.ufrgs.br, letícia@eletro.ufrgs.br\} 
method obtains a correct iris recognition rate of $94,68 \%$, even considering out of focus images and iris occlusions; a correct iris recognition rate of $100 \%$ is obtained excluding problematic images. The proposed method is flexible, and allows to fine tune the iris recognition criterion according to the accuracy level required by the application.

\section{Introdução}

Para controlar o acesso a áreas de segurança, é necessária uma infra-estrutura confiável para a identificação pessoal. Métodos convencionais de identificação, usando senhas e cartões, não são considerados por especialista como "altamente confiáveis", pois senhas e cartões podem ser esquecidos ou furtados [1]. A tecnologia biométrica, baseada na identificação de características físicas ou comportamentais dos humanos, tais como rostos (faces), impressões digitais, formato das mãos, olhos, assinaturas ou mesmo voz, tem se mostrado uma alternativa viável aos sistemas de controle de acesso atualmente existentes em diversas aplicações [1].

Entre as diversas características físicas utilizadas, a íris têm atraído muita atenção nas últimas décadas na tecnologia biométrica. O uso da íris como método biométrico foi sugerido originalmente pelos oftalmologistas Adler e Rohen na década de 60 [2], e também por Flom e Safir em meados dos anos 80 [3], em cujos trabalhos confirmaram que a estrutura da íris de um indivíduo é única e estável ao longo da sua vida. Outra grande vantagem da íris na identificação pessoal é que esta parte do olho é claramente visível (ao invés da retina, por exemplo), facilitando a aquisição de imagens de texturas da íris [4].

No campo de pesquisa de reconhecimento de texturas, inúmeras técnicas de filtragem multicanal têm sido aplicadas, tais como: Transformada de Gabor [2] [4] [5], Transformadas Wavelets unidimensionais e bidimensionais [6], Transformada Wavelet Packet [7], Transformada de Hilbert 2D [8], Pirâmide Laplaciana [9] e Análise de Componentes Independentes [10].

Utilizando-se os filtros de Gabor, pode-se calcular a Transformada de Gabor através da abordagem de Transformada Wavelet [11][12][14], aqui denominada de Transformada Wavelet Gabor (TWG), e seguindo esta abordagem, é demonstrado que esta transformada tem propriedades que a tornam atrativa em aplicações de visão computacional, tais como a sua simplicidade e ótima localização nos domínios espaço e freqüência. Através da TWG pode-se representar alguns dos aspectos visuais das texturas, tais como a orientação local da textura, ou ainda a caracterização do padrão espacial da textura, com base nas informações contidas em diferentes sub-bandas do domínio freqüência [11].

Apesar das boas características da Transformada de Gabor e da Transformada Wavelet Gabor, estas apresentam como problema a redundância de informações no domínio da transformada. Manjunah e Ma apresentaram uma maneira de escolher as bases da Transformada de Gabor de forma que esta redundância de informações seja minimizada [12]. Esta transformada é aqui denominada de Transformada Wavelet de Gabor Ótima (TWGO), e foi proposta tendo em vista a sua aplicação na segmentação de imagens de texturas. 
Neste trabalho é então proposto o uso da Transformada Wavelet de Gabor Ótima (TWGO) para a extração de características de imagens de textura da íris. A técnica apresentada neste trabalho usa um banco de 24 filtros de Gabor "sem redundância" construído conforme [12]. Por fim, é feita a avaliação desta nova aplicação da técnica desenvolvida em [12] para o campo de reconhecimento de íris.

Este artigo está organizado da seguinte forma: a Seção 2 apresenta uma descrição detalhada do método proposto para o reconhecimento de íris. Os experimentos e resultados são mostrados na Seção 3. Finalmente, a Seção 4 apresenta as conclusões deste artigo.

\section{Reconhecimento da Íris}

Sistemas de reconhecimento de íris normalmente são organizados em módulos (ou também etapas do processamento), definidos por: (1) Pré-processamento da imagem, composto por segmentação localização da íris na imagem e sua normalização; (2) extração de características e (3) classificação de imagens de íris. Uma descrição detalhada destas etapas é apresentada a seguir.

\subsection{Pré-processamento das imagens de íris}

Freqüentemente, as imagens de íris incluem mais informações do que apenas a região de interesse (íris). A Figura 1 ilustra uma imagem típica, que é também composta de partes do olho que não são usadas no reconhecimento de íris, como: pupila, cílios, esclera e pálpebras. Sendo assim, faz-se necessário um pré-processamento da imagem para localizar a íris, antes da etapa de extração de características.

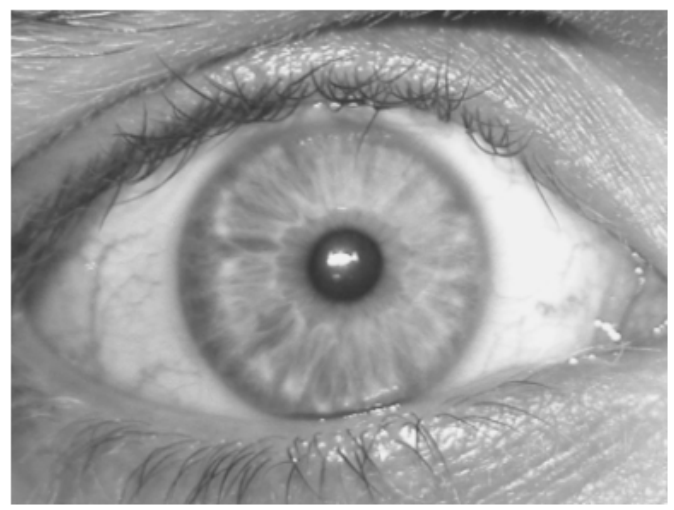

Figura 1. Imagem típica do banco de íris UBIRIS. 


\subsubsection{Localização da Íris}

Dada uma imagem do olho em tons de cinza $I(x, y)$, é necessário fazer a localização da íris dentro desta imagem, detectando as bordas internas e externas da íris e seu centro. Um operador integral-diferencial, como sugerido em [2] pode ser utilizado para encontrar estas bordas circulares. Primeiramente é calculada a expressão de transformação da imagem $I(x, y)$ através da integral de contorno dada por:

$$
T\left(r, x_{0}, y_{0}\right)=\left|\oint_{r} \frac{\partial}{\partial r}\left(\frac{G_{\sigma}(r) * I(s)}{2 \pi r}\right) d s\right|,
$$

onde $G_{\sigma}$ é a função Gaussiana de suavização com escala $\sigma ; r$ é o raio; ‘*’ denota a operação de convolução, e $I(s)$ denota a intensidade de um pixel no elemento $\mathrm{S}$ do círculo de raio $r \mathrm{e}$ centrado em $x_{0}$ e $y_{0}$.

Após, busca-se na função $T\left(r, x_{0}, y_{0}\right)$ os seus valores de máximo global. Associado a cada máximo global desta função corresponderá a um círculo na imagem centrado nas coordenadas $x_{0}$ ' e $y_{0}$ ' e com um raio $r$ '. Como a imagem provavelmente conterá apenas 2 círculos pronunciados, correspondentes ao limite da íris com a esclera e à pupila, estes círculos estarão associados aos 2 primeiros máximos de $T\left(r, x_{0}, y_{0}\right)$. Neste trabalho, uma vez que as imagens do banco são similares, para otimizar este algoritmo restringiu-se a área de pesquisa na imagem a uma região específica de valores $x_{0}$ no intervalo $\left[200,350\right.$ ] e $y_{0}$ no intervalo [300,500].

\subsubsection{Normalização do Tamanho da Imagem: Retificação da Íris}

Como as imagens podem ser capturadas sob diferentes condições (por exemplo, com dilatação da pupila, movimentação do olho, inclinação da cabeça, etc.), após a localização dos limites da íris obtém-se uma representação retificada da imagem para que imagens diferentes possam ser processadas e comparadas da mesma maneira (isto é, as imagens passam a ser representadas da mesma forma, com o mesmo número de pixels). Esta retificação é obtida mapeando os valores dos pixels da imagem da íris originalmente em coordenadas cartesianas $I(x, y)$ para um sistema de coordenadas polares $I(x(r, \theta) y(r, \theta))$ (redefinido como $I(r, \theta)$ ) [2]. As coordenadas $x(r, \theta)$ e $y(r, \theta)$ deste novo mapeamento são dadas por:

$$
\begin{aligned}
& x(r, \theta)=(1-r) x_{p}(\theta)+r x_{s}(\theta) \\
& y(r, \theta)=(1-r) y_{p}(\theta)+r y_{s}(\theta)
\end{aligned}
$$

onde o raio $r$ está no intervalo de [0,1]; o ângulo $\theta$ está entre $[0,2 \pi] ;\left(x_{p}(\theta), y_{p}(\theta)\right)$ são os pixels ao longo da borda interna da íris e $\left(x_{s}(\theta), y_{s}(\theta)\right)$ são os pixels ao longo da borda externa da íris [2]. Este processo de retificação da forma circular da íris é também chamado de 
normalização, pois mapeia a imagem original da íris usando $N_{r}$ raios e $N_{\theta}$ ângulos, formando uma imagem retificada com tamanho normalizado de $\left(N_{r} \times N_{\theta}\right)$ pixels. Neste trabalho foram usados $N_{r}=64$ e $N_{\theta}=512$. O resultado desta transformação está ilustrado na Figura 2.

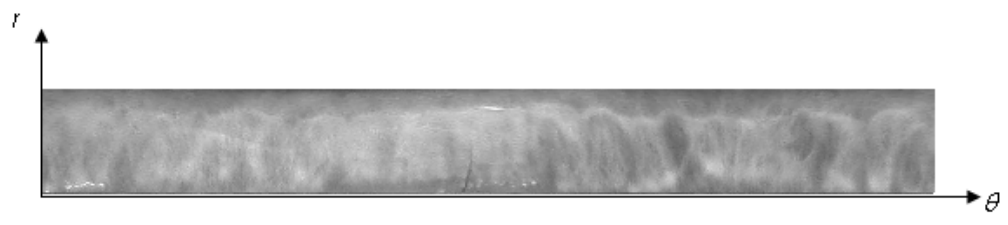

Figura 2. Imagem da figura 1 normalizada.

\subsection{Extração das Características}

Nesta etapa é feita a extração das características relevantes da imagem de íris, representado-a através de um conjunto reduzido de dados (feições). Uma abordagem usualmente adotada é a de usar transformações da imagem, que geram novos espaços de representação, a partir dos quais se obtêm as estatísticas que formam o vetor de características. Neste trabalho foi utilizada a Transformada Wavelet Gabor Ótima. A sua definição, bem como os motivos que nos levaram a usar esta transformada são explicados a seguir.

\subsubsection{Função Gabor Bidimensional}

O conjunto original de filtros proposto por Gabor, estendido para o caso bidimensional [2], leva a definição da função de Gabor bidimensional $g(x, y)$ descrita como:

$$
g(x, y)=\left(\frac{1}{2 \pi \sigma_{x} \sigma_{y}}\right) \exp \left[-\frac{1}{2}\left(\frac{x^{2}}{\sigma_{x}^{2}}+\frac{y^{2}}{\sigma_{y}^{2}}\right)+j 2 \pi W x\right],
$$

onde $\sigma_{x}$ e $\sigma_{y}$ são os desvios padrões nos eixos $x$ e $y$, respectivamente, e $W$ é a freqüência do filtro.

Através da variação de $\sigma_{x}, \sigma_{y}$ e $W$ obtêm-se uma família de funções (também denominada de base de representação ou filtros de Gabor) e pode-se decompor um sinal (ou imagem) sobre esta base, através de uma transformação integral denominada de Transformada Gabor 2-D. Desta forma, obtêm-se uma representação conjunta de informações disponíveis nos domínios espaço e de freqüência. Entretanto observa-se que as funções de Gabor formam um conjunto de bases que, apesar de poder ser completo, não necessariamente é ortogonal [2]. Isto significa que uma transformação integral usando estas funções como base poderá ter uma alta redundância de informações [12]. 
Uma outra família de funções auto-similares pode ser gerada a partir da $g(x, y)$, sendo denominada de Wavelets de Gabor. Esta nova classe pode ser gerada por [12]:

$$
\begin{gathered}
g_{m n}(x, y)=a^{-m} g\left(x^{\prime}, y^{\prime}\right), a>1 ; m, n \in \mathrm{N}^{*}, \\
x^{\prime}=a^{-m}(x \cos \theta+y \sin \theta), \\
y^{\prime}=a^{-m}(-x \sin \theta+y \cos \theta), \\
\theta=n \pi / K,
\end{gathered}
$$

onde $K$ é o número total de orientações; $S$ o número total de escalas das funções Gabor; $a$ é a constante de passo da escala; $m$ é a escala, $m=0,1, \ldots, S$ - 1 ; e $n$ é o indexador das orientações, $n=0,1, \ldots, K-1$. O parâmetro de escala $a^{-m}$ tem o objetivo de assegurar que a energia seja independente da escala $m$. Neste trabalho foi usado um passo de escala de 2, correspondendo às transformações diádicas [13].

A não-ortogonalidade da família de Wavelets de Gabor ocasiona a redundância de informação nas transformadas obtidas. Por isto, Manjunath e Ma [12] definem uma estratégia para reduzir esta redundância. Sendo $K$ o número desejado de orientações e $S$ o número desejado de escalas na decomposição em múltiplas escalas, os autores propõem um algoritmo para o cálculo de $\sigma_{x}, \sigma_{y}$ e $W$ que assegurem que as respostas em frequiência desta família de filtros se tangenciem apenas quando a magnitude das respostas cai à metade dos seus respectivos valores de pico, minimizando assim a sua sobreposição no domínio freqüência. Esta estratégia é expressa pelas seguintes equações:

$$
\begin{gathered}
a=\left(U_{h} / U_{l}\right)^{\frac{1}{S-1}}, \\
\sigma_{x}=\frac{(a-1) U_{h}}{(a+1) \sqrt{2 \ln 2}} \\
\sigma_{y}=\tan \left(\frac{\pi}{2 K}\right)\left[U_{h}-2 \ln \left(\frac{2 \sigma_{x}^{2}}{U_{h}}\right)\right]\left[2 \ln 2-\frac{(2 \ln 2)^{2} \sigma_{x}^{2}}{U_{h}^{2}}\right]^{-} \\
, \quad \\
W=U_{h},
\end{gathered}
$$

onde $a$ é uma variável a ser usada no cálculo de $\sigma_{x}, U_{l}$ e $U_{h}$, são os centros das freqüências inferior e superior desejadas para os filtros de Gabor, respectivamente. $\sigma_{x}$ e $\sigma_{y}$ são as larguras das janelas calculadas e $W$ é a freqüência da função Gabor mãe que gerará por (5) (6), (7) e (8) os filtros com o mínimo de superposição no domínio freqüência. 
O banco de (K x S) filtros gerado da forma acima descrita é chamado Banco de Filtros Ótimos de Gabor ou somente Filtros Ótimos de Gabor (FOG). Estes filtros podem então ser utilizados em uma transformação integral (descrita na Equação 13, a seguir), denominada de Transformada Wavelet de Gabor Ótima (TWGO).

Neste trabalho foram usados os parâmetros $U_{h}=0.4, U_{l}=0.05, K=6$ e $S=4$ [12] nas equações (9), (10) (11) e (12), e com os resultados obtidos para $\sigma_{x}, \sigma_{y}$ e $W$ nas equações (5), (6), (7) e (8), para se gerar um banco de 24 filtros FOG. Uma representação destes filtros pode ser vista na Figura 3, onde o contorno indica quando a magnitude das respostas dos filtros cai à metade dos seus respectivos valores de pico.

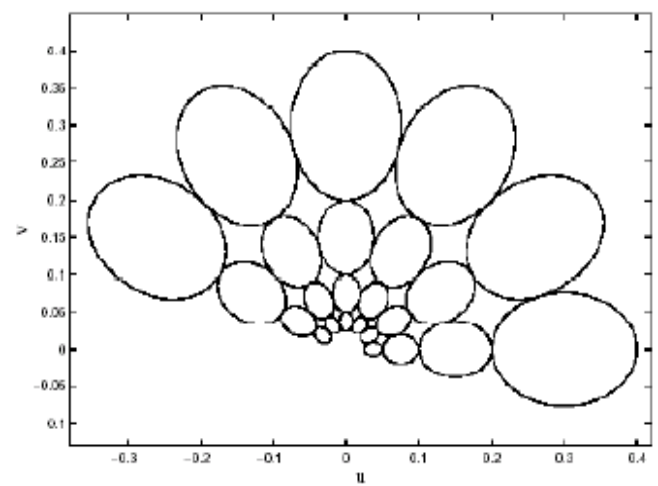

Figura 3. Representação dos 24 filtros Gabor com redundância mínima, utilizando $U_{h}=0.4$, $\mathrm{U}_{\mathrm{l}}=0.05, \mathrm{~K}=6$ e $\mathrm{S}=4[12]$.

Assim, dada uma imagem $I(x, y)$ e a família de Wavelets Gabor geradas por (9), (10), (11) e (12) e pelas equações (5), (6), (7) e (8), a Transformada Wavelet de Gabor Ótima (TWGO) é definida por:

$$
H_{m n}(x, y)=I(x, y) * g_{m n}(x, y)=\iint I\left(x_{1,} y_{1}\right) g_{m n}\left(x-x_{1}, y-y_{1}\right) d x_{1} d y_{1},
$$

onde $H_{m n}(x, y)$ é a imagem filtrada pelo filtro de Gabor $g_{m n}$ com escala $m$ e orientação $n$, e '*' indica o operador de convolução.

Neste trabalho, a imagem normalizada é dividida em sub-imagens, ou blocos $B_{b}$ $b=1,2, \ldots, N_{B}$. Nos experimentos, foram testados diferentes tamanhos de blocos para se verificar a capacidade de discernimento entre micro-texturas locais na área da íris. Os tamanhos de blocos usados foram: 8x8, 16x16, 32x32 e 64x64. Então a TWGO é calculada para cada um destes blocos em separado, sendo que os filtros de Gabor têm as mesmas dimensões dos blocos e usando-se os mesmos parâmetros sugeridos em [12] $\left(U_{h}=0.4\right.$, $U_{l}=0.05, K=6$ e $\left.S=4\right)$. Isto resulta em 24 respostas $H_{m n}(x, y)$ para cada bloco da imagem. 


\subsubsection{Vetor de Características}

Freqüência, orientação e complexidade são algumas das feições que podem ser utilizadas para discriminar texturas de forma compatível com a percepção visual [11]. Freqüência e orientação são feições implícitas na própria resposta dos filtros da TWGO, e podem ser representadas pela média da resposta de cada filtro aplicado a cada bloco da imagem. $\mathrm{O}$ desvio padrão da resposta do filtro no bloco $\mathrm{B}_{\mathrm{b}}$ representa a complexidade local da textura, portanto esta feição pode ser usada como uma característica adicional, para melhorar a taxa de acerto na classificação das texturas em relação àquela que seria obtida se fosse usado apenas o vetor de médias [11].

Sendo $H_{m n}(x, y)$ a resposta para cada bloco $B_{b}$ do filtro de Gabor com uma determinada freqüência e orientação, usou-se a magnitude de $H_{m n}(x, y)$ para analisar as respostas obtidas [14]. Assim, tomando-se estas 24 respostas de filtros para um bloco $B_{b}$, representamos a informação local da textura neste bloco através de parâmetros estatísticos calculados para $H_{m n}(x, y)$. Os dois parâmetros estatísticos mais usados na literatura são a média e o desvio padrão [12] [14], sendo a média da magnitude da resposta do bloco calculada por:

$$
\mu_{b}=\frac{1}{M N} \sum_{x=1}^{M} \sum_{y=1}^{N}\left|H_{m n}(x, y)\right|
$$

onde $\mu_{b}$ é a média do bloco $B_{b}, M$ e $N$ são as dimensões do bloco nos eixos $x$ e $y$, para $x=1,2, \ldots, M$ e $y=1,2, \ldots, N$.

O desvio padrão também pode ser utilizado como característica adicional, que complementa as características existentes [12], sendo dado por:

$$
\sigma_{b}=\sqrt{\frac{1}{M N} \sum_{x=1}^{M} \sum_{y=1}^{N}\left(\left|H_{m n}(x, y)\right|-\mu_{b}\right)^{2}}
$$

onde $\sigma_{b}$ é o desvio padrão e $\mu_{b}$ é a média definida na equação (14).

Tendo os dois momentos estatísticos, média $\mu_{b}$ e desvio padrão $\sigma_{b}$, pode-se estimar a energia total de cada bloco $B_{b}$ através de:

$$
e_{b}=\sqrt{\mu_{b}^{2}+\sigma_{b}^{2}}
$$

Dados os momentos estatísticos descritos nas equações (14), (15) e (16), a imagem pode ser representada de diferentes formas, através de um vetor de médias $\vec{V}_{\mu}$, um vetor de 
desvios padrões $\vec{V}_{\sigma}$, um vetor de energias totais $\vec{V}_{e}$, ou ainda por um quarto vetor de características $\vec{V}_{\mu \sigma}$ formado pela concatenação dos vetores de média e desvio padrão, definido por:

$$
\vec{V}_{\mu \sigma}=\left\{\vec{V}_{\mu}, \vec{V}_{\sigma}\right\}
$$

onde $\vec{V}_{\mu \sigma}$ é o vetor de características resultante, $\vec{V}_{\mu}$ é o vetor de características de média e $\vec{V}_{\sigma}$ é o vetor de características de desvio padrão. Estas representações serão comparadas na seção que descreve os nossos experimentos.

\subsection{Comparação entre Representações das Imagens de Íris}

Após a extração de características da imagem normalizada da íris e da obtenção de um dos 4 possíveis tipos de vetor de características, então faz-se o reconhecimento da íris medindo a distância (ou dissimilaridade) entre o vetor de características da íris de entrada, e os vetores de características dos protótipos das classes do problema.

Existem diversas formas de medir a distância entre dois vetores. Neste trabalho foram usadas duas medidas: Soma das Diferenças Absolutas, também conhecida como city block [15] e a Distância Euclidiana Modificada. Assim, dados dois vetores $p$ e $q$, a soma das diferenças absolutas $d(p, q)$ e a distância Euclidiana modificada $d e(p, q)$ são respectivamente expressas por :

$$
\begin{gathered}
d(p, q)=\sum_{i=1}^{N}\left|p_{i}-q_{i}\right| \\
d e(p, q)=\sqrt{\sum_{i=1}^{N} \frac{\left(p_{i}-q_{i}\right)^{2}}{\sigma_{p} \cdot \sigma_{q}}},
\end{gathered}
$$

onde $p_{i}$ é a i-ésima característica da íris de entrada, $q_{i}$ é a i-ésima característica da íris usada para comparação (armazenada), $\sigma_{p}$ e $\sigma_{q}$ são os desvios padrões das características em $p$ e $q$, respectivamente, e $N$ é o número de elementos em cada um dos vetores de características em $p$ e $q$. Quanto menor a distância entre duas amostras, representadas pelos vetores de características $p$ e $q$, maior será a similaridade entre estes vetores.

Para o caso do cálculo das distâncias envolvendo o vetor de características $\vec{V}_{\mu \sigma}$, normalizou-se as medidas de modo similar ao apresentado em [12], dividindo-se o vetor 
$\vec{V}_{\mu}$, pela média de todas características da base dados e também o vetor $\vec{V}_{\sigma}$ pela variância de todas características da base de dados.

\section{Experimentos}

Este artigo apresenta um método para a extração de características da íris e para a identificação de imagens da íris. Foram realizados diversos experimentos para avaliar o método proposto e compará-lo com outros métodos, que serão reportados a seguir. A implementação dos métodos foi realizada em Matlab 5.3 para Windows, em um computador do tipo IBM PC com processador AMD Athlon 64 3200+, clock de 2,01 GHz, e 1GB de memória RAM.

\subsection{Banco de Imagens}

O banco de imagens de íris usado nos experimentos é denominado UBIRIS, criado pelo departamento de informática da Universidade de Beira Interior, Portugal [16]. Este banco de imagens é composto por 1877 imagens de 241 pessoas. Cada imagem tem resolução 800 x 600 pixels, e foi armazenada no formato JPEG.

O objetivo deste banco de íris é o fornecimento de imagens com diferentes tipos de ruído, simulando a captura da imagem em boas e más condições, permitindo o desenvolvimento e a avaliação de metodologias robustas para a identificação da íris.

As imagens foram adquiridas em duas etapas. Na primeira etapa da captura das imagens, fatores que geram artefatos nas imagens foram minimizados, especialmente aqueles relativos a reflexos, luminosidade e contraste, fazendo-se que a aquisição de imagens ocorresse em uma sala escura. Uma imagem capturada nesta etapa é mostrada na figura 1 . Na segunda etapa, a posição da câmera foi modificada a fim de introduzir alterações de luminosidade, contraste e foco, assim como reflexos. As imagens utilizadas nos nossos experimentos são referentes à primeira etapa, sendo 5 imagens de cada íris, totalizando 1205 imagens. Deste grupo foram então selecionadas 500 imagens de 100 pessoas (5 imagens para cada pessoa) por não possuírem uma íris com regiões importantes oclusas por pálpebras ou cílios, ou até mesmo imagens de olhos fechados e com artefatos severos de iluminação e foco. Este grupo é utilizado para validar a técnica proposta, e para avaliar quais opções de tamanho de bloco e de vetor de características, assim como qual medida de dissimilaridade, deveriam ser usadas para obter os melhores resultados. Uma vez verificadas as melhores opções, estas são então aplicadas às outras imagens, para avaliar a robustez do algoritmo aos defeitos de aquisição da imagem. 


\subsection{Resultados Experimentais do Reconhecimento de Íris}

A Tabela 1 apresenta as dimensões dos vetores de características obtidos com diferentes tamanhos de blocos, usando como descritores da imagem $\vec{V}_{\mu \sigma}, \vec{V}_{\mu}$ ou $\vec{V}_{e}$, ou seja, média e desvio padrão concatenados, média ou energia total, como descritores estatísticos de cada bloco da imagem. Note que o vetor de características $\vec{V}_{\sigma}$ não é utilizado independentemente, mas como uma feição adicional para aumentar a robustez da classificação. Isto se deve a observação de Manjunath e Ma [12] que a classificação de imagens de íris pode ser mais robusta se os desvios padrões em conjunto com as médias, ao invés de usar médias ou desvios padrões de forma independente.

Tabela 1. Dimensões dos vetores de características.

\begin{tabular}{ccccc}
\hline Características & 8 X8 & 16 X16 & 32 X32 & 64 X64 \\
\hline Média e Desvio Padrão Concatenados & 24576 & 6144 & 1536 & 384 \\
Média ou Energia & 12288 & 3072 & 768 & 192 \\
\hline
\end{tabular}

\subsubsection{Resultados do Reconhecimento}

Para comparar os vetores de características da íris de entrada com os armazenados, foram utilizadas as distâncias city block e Euclidiana Ponderada Modificada. A dissimilaridade pela city block foi calculada para $\vec{V}_{\mu \sigma}, \vec{V}_{\mu}$ e $\vec{V}_{e}$. Abaixo, estão listadas as medidas de dissimilaridade entre vetores que foram testadas em nossos experimentos:

1. City Block de $\vec{V}_{\mu \sigma}$;

2. City Block de $\vec{V}_{\mu}$;

3. City Block de $\vec{V}_{e}$;

4. Distância Euclidiana Modificada de $\vec{V}_{\mu \sigma}$;

5. Distância Euclidiana Modificada de $\vec{V}_{e}$. 
Uma imagem de entrada é comparada com as imagens armazenadas com base na máxima similaridade, ou seja, a íris de entrada é atribuída à classe de uma das íris armazenadas pela menor distância entre seus vetores de características. Se as imagens pertencem a mesma pessoa, houve um acerto, no caso contrário houve um erro.

Os resultados percentuais de acerto para os diferentes vetores de características estão expostos na Tabela 2, para as 500 íris selecionadas, e usando o vetor de características da TWGO. O mesmo método é aplicado para o restante das imagens (705 imagens), e seus resultados são mostrados na Tabela 3. Os resultados para o banco de imagens completo (1205 imagens) são apresentados na Tabela 4.

Com relação aos itens 4 e 5 acima, com base nos resultados dos itens 1,2 e 3 apresentados nas tabelas 2 e 3 , observou-se que os melhores resultados obtidos com a métrica city block são os vetores de características $\vec{V}_{\mu \sigma} \vec{V}_{e}$, sendo então que se optou por aplicar a métrica distância Euclidiana Modificada apenas para estes dois vetores, para fins de comparação.

Tabela 2. Percentuais de acerto na identificação da íris (500 imagens).

\begin{tabular}{ccccc}
\hline Método & $8 \times 8$ & $16 \times 16$ & $32 \times 32$ & $64 X 64$ \\
\hline 1 & 100 & 100 & 100 & 98,4 \\
2 & 100 & 100 & 99,8 & 95,8 \\
3 & 100 & 100 & 99,8 & 96 \\
4 & 99,4 & 99,2 & 98,2 & 96,8 \\
5 & 99,4 & 98,8 & 98,4 & 94,8 \\
\hline
\end{tabular}

Tabela 3. Percentuais de acerto na identificação da íris (705 imagens).

\begin{tabular}{|ccccc|}
\hline Método & $8 \times 8$ & $16 \times 16$ & $32 \times 32$ & $64 X 64$ \\
\hline 1 & 90,922 & 90,0709 & 86,2411 & 78,5816 \\
2 & 90,7801 & 89,9291 & 85,2482 & 70,9220 \\
3 & 91,0638 & 89,9291 & 85,1064 & 73,3333 \\
4 & 84,1135 & 83,1206 & 80,7092 & 71,773 \\
5 & 83,9716 & 82,8369 & 78,156 & 67,5177 \\
\hline
\end{tabular}


Tabela 4. Percentuais de acerto na identificação da íris (1205 imagens).

\begin{tabular}{|ccccc|}
\hline Método & $8 \times 8$ & $16 \times 16$ & $32 \times 32$ & $64 X 64$ \\
\hline 1 & 94,68 & 94,19 & 91,95 & 87,46 \\
2 & 94,60 & 94,10 & 91,36 & 82,98 \\
3 & 94,77 & 94,10 & 91,28 & 84,4 \\
4 & 90,70 & 90,12 & 88,71 & 83,48 \\
5 & 90,62 & 89,95 & 87,2 & 81 \\
\hline
\end{tabular}

\subsubsection{Discussão e Comparação com Métodos Existentes}

Inicialmente o método proposto neste trabalho foi testado nas 500 imagens de íris selecionadas. Estas imagens não possuem degradações devido a foco incorreto, iluminação, olhos fechados e outras formas de degradações das imagens de íris. As distâncias city block e Euclidiana Modificada foram utilizadas para os cálculos de dissimilaridade entre os vetores de características da íris. Observando a Tabela 2, nota-se que diversas opções testadas conseguiram obter uma classificação de $100 \%$ de acerto por distância mínima. A distância city block gerou os melhores resultados como pode ser visto na Tabela 2. Isto está de acordo com os resultados obtidos em [11], onde são relatadas diferentes utilizações da Transformada de Gabor para classificação de texturas. Pode-se notar também que os métodos apresentam resultados similares com uso de blocos no tamanho 8x8 e 16x16. Assim, para se verificar quais opções de tamanho bloco, vetor de características e medidas de distância seriam mais eficientes, aplicou-se as mesmas técnicas no resto das imagens, cujos resultados são os mostrados nas Tabelas 3 e 4. Neste caso, o uso da distância city block aplicada nos vetores de características de energia total com blocos $8 \times 8$ foi o método que apresentou melhor resultado, gerando uma taxa de acerto de 91,06\% (para 705 imagens) ou 94,77\%(para 1205 imagens). A Tabela 5 apresenta a comparação desta melhor taxa de acerto com resultados obtidos por outros autores.

Tabela 5. Comparação dos resultados entre diferentes métodos de extração de característica da íris utilizando a TWGO.

\begin{tabular}{|cc|}
\hline Autor & Resultado em taxa de acerto \\
\hline $\begin{array}{c}\text { Método proposto } \\
\text { (500 imagens) }\end{array}$ & $100 \%$ \\
\hline $\begin{array}{c}\text { Método proposto } \\
\text { (705 imagens) }\end{array}$ & $91,06 \%$ \\
\hline $\begin{array}{c}\text { Daugman [2] } \\
\text { Ma [4] }\end{array}$ & $100 \%$ \\
Zhu [5] & $99.85 \%$ \\
\hline
\end{tabular}


Assim, comparando-se os resultados obtidos pela técnica aqui descrita com aquelas propostas por outros autores, nota-se que quando as imagens forem previamente selecionadas, a técnica aqui proposta atinge os 100\% de acerto, equiparando-se com aos resultados obtidos por Daugman [2], cujo método também seleciona apenas imagens de íris que não contenham erros de aquisição, por isto atinge a elevada taxa de acerto mostrada. Em Ma et al. [4], existe também um pré-processamento para eliminar partes defeituosas na imagem adquirida, e em Zhu et al. [5] é feito uma equalização histograma para corrigir o efeito da iluminação não homogênea (embora não exista nenhum detalhamento sobre a qualidade das imagens adquiridas pelo sistema proposto).

Quando se aplica a técnica ao resto do banco de imagens, neste caso o melhor acerto obtido por nossa técnica foi de $91,06 \%$ (considerando-se somente as 705 imagens), ou foi $94,77 \%$ considerando-se o banco inteiro de imagens. Isto significa que 63 imagens de íris não foram corretamente associadas ao indivíduo adequado. Esta taxa é um pouco mais baixa que a obtida pelos outros autores citados [4] [5]. Entretanto, observando-se estas 63 imagens que deram problemas, nota-se que elas contêm algum grave defeito de aquisição (oclusão do olho, reflexos muito intensos sobre a área da íris, olhar na direção errada). Assim, se o método deste artigo descartasse estas imagens, obter-se-ia uma taxa idêntica aquela reportada em [2], e superior às obtidas em [4] e [5].

Outros trabalhos de reconhecimento de íris também fazem o uso da Transformada de Gabor para gerar o vetor de características da íris, porém com uma abordagem diferente da apresentada neste trabalho. Daugman [2] utiliza uma outra abordagem para os filtros Gabor, na qual a representação da imagem é feita através de coeficientes de um conjunto completo de filtros Gabor, gerando códigos de íris na mesma dimensão da íris normalizada. Apesar de obter um vetor de características bastante reduzido, o cálculo da transformada é feito de forma muito custosa [17]. Em [5] os autores abordam o uso dos filtros de Gabor, com 4 orientações e 6 escalas, mas não apresenta os desvios padrões dos filtros para se ter certeza de que estes não possuem redundância. Um outro filtro de Gabor é usado em [4], porém difere do apresentado neste trabalho por não possuir orientação, este filtro é denominado de Filtro Simétrico Circular. O método proposto neste trabalho difere dos demais pelo uso do banco de filtros de Gabor escolhido para fazer a extração das características, ou seja, fazemos uso da TWGO [12] para representar e classificar as texturas de íris, obtendo respostas da transformação sem redundância, com melhor capacidade de representação da textura. 


\section{Conclusões}

Este artigo investiga a aplicação da TWGO para representar e classificar imagens de íris. Os resultados experimentais obtidos indicam que este método potencialmente pode ser eficiente para discriminar entre as imagens de uma mesma íris e de íris diferentes. Com o método proposto, são obtidas taxas de classificação correta de imagens da íris compatíveis com o estado da arte, e em alguns casos até superiores aquelas obtidas por outros métodos recentemente propostos na literatura, com uma representação menos redundante, que também é robusta a (pequenas) alterações da íris ocorridas durante a aquisição das imagens.

Sugere-se como prosseguimento deste trabalho a investigação da distância de Bhattacharya para o vetor de características contendo média e desvio-padrão concatenados. Uma vez que os filtros ótimos de Gabor produzem uma resposta máxima quando sua oscilação e rotação "casam" com a textura do bloco da imagem a ser analisado, também sugere-se investigar e avaliar o uso de um vetor de características que contenha apenas esta melhor resposta por bloco, ao invés dos 24 respostas obtidas. Por fim uma característica importante da íris que também pode ser explorada é a cor. A incorporação desta característica poderia melhorar a classificação das imagens de íris, dado que a íris é colorida.

\section{Referências}

[1] Woodward, J. D., "Biometrics: Privacy's Foe or Privacy's Friend?" IEEE Proceedings of the Institute of Electrical and Electronics Engineers, v. 85, n. 9, p. 1480-1492, sep. 1997.

[2] Daugman J., "High confidence visual recognition of person by a test of statistical independence", IEEE Transactions on Pattern Analysis and Machine Intelligence, 1993, pp. 1148-1161.

[3] Flom L. , A. Safir, Patent Nº.4641349, U.S. Government Printing Office Washington, DC, 1987.

[4] Ma L. , Y. Wang, T. Tan, "Iris recognition using circular symmetric filters". National Laboratory of Pattern Recognition, Institute of Automation, Chinese Academy of Sciences, 2002.

[5] Zhu Y., T. Tan, Y. Wang, "Biometric Personal Identification Based on Iris Patterns", 15th International Conference on Pattern Recognition, 2000 , pp. 801 -804.

[6] Boles, W.W, B. Boashash, "A Human Identification Technique Using Images of the Iris and Wavelet Transform”. IEEE Transaction on signal processing, v. 46, n. 4, p. 1185-1188, 1998

[7] Rossant, F. et al., "Iris identification and robustness evaluation of a wavelet packets based algorithm". IEEE International Conference on Image Processing. ICIP 2005, p. 257-260, 2005.

[8] Tisse, C. et al., "Person identification technique using human iris recognition". IEEE Transactions on Systems, Man and Cybernetics, Part C: Applications and Reviews, p. 435- 441, 2002.

[9] Wildes, R, "Iris Recognition: An Emerging Biometric Technology". Proceedings of the IEEE, v. 85, n. 9, p. 1348-1363, 1997. 
[10] Huang, Y.P., S.W. Luo, E.Y. Chen, “An Efficient Iris Recognition System”. First International Conference on Machine Learning and Cybernetics, p. 450-454, 2002.

[11] Clausi D., M. Jernigan , "Designing Gabor Filters for Optimal Texture Separability”, Pattern Recognition, 2000, pp. 1835-1849.

[12] Manjunath B., W. Ma, “Texture Feature for Browsing and Retrieval of Image Data”, IEEE Transaction on Pattern Analysis and Machine Intelligence, 1996, pp. 837-842.

[13] Mallat S., S. Zhong, "Characterization of signal from multiscale edges, IEEE Transactions on Pattern Analysis and Machine Intelligence, 1992, pp. 710-732.

[14] Bovik, A.C., M. Clark; W.S. Geisler, "Multichannel texture analysis using localized spatial." IEEE Transactions Pattern Analysis and Machine Intelligence, 1990, pp. 55-73.

[15] Gonzalez, R. C., R. E. Woods, "Digital Image Processing”, Addison Wesley, 1992.

[16] Proença H., L. A. Alexandre, "UBIRIS: A noisy iris image database", Proceed. of ICIAP 2005 - International Conference on Image Analysis and Processing, 2005, pp. 970-97.

[17] Daugman J. G. , "Complete Discrete 2D Gabor Transforms by neural networks for Image Analysis and Compression”, IEEE Transactions on Acoustics, Speech and Signal Processing, 1988, v. 36, pp. 1169-1179. 